\title{
Tunable optical delay line based on integrated grating-assisted contradirectional
} couplers

\author{
Xu, Wang; Yuhe, Zhao; Ding, Yunhong; Xiao, Sanshui ; Dong, Jianji
}

Published in:

Photonics Research

Link to article, DOI:

10.1364/PRJ.6.000880

Publication date:

2018

Document Version

Publisher's PDF, also known as Version of record

Link back to DTU Orbit

Citation (APA):

Xu, W., Yuhe, Z., Ding, Y., Xiao, S., \& Dong, J. (2018). Tunable optical delay line based on integrated gratingassisted contradirectional couplers. Photonics Research, 6(9), 880-86. https://doi.org/10.1364/PRJ.6.000880

\section{General rights}

Copyright and moral rights for the publications made accessible in the public portal are retained by the authors and/or other copyright owners and it is a condition of accessing publications that users recognise and abide by the legal requirements associated with these rights.

- Users may download and print one copy of any publication from the public portal for the purpose of private study or research.

- You may not further distribute the material or use it for any profit-making activity or commercial gain

- You may freely distribute the URL identifying the publication in the public portal 


\title{
PHOTONICS Research
}

\section{Tunable optical delay line based on integrated grating-assisted contradirectional couplers}

\author{
Xu Wang, ${ }^{1}$ Yuhe Zhao, ${ }^{1}$ Yunhong Ding, ${ }^{2}$ Sanshui Xiao, ${ }^{2}$ (i) and Jianji Dong ${ }^{1, *}$ (i) \\ ${ }^{1}$ National Laboratory for Optoelectronics, School of Optical and Electronic Information, Huazhong University of Science and Technology, \\ Wuhan 430074, China \\ ${ }^{2}$ Department of Photonics Engineering, Technical University of Denmark, 2800 Kgs. Lyngby, Denmark \\ *Corresponding author: jjdong@mail.hust.edu.cn
}

Received 27 May 2018; revised 22 July 2018; accepted 24 July 2018; posted 25 July 2018 (Doc. ID 332640); published 21 August 2018

\begin{abstract}
Tunable optical delay lines are one of the key building blocks in optical communication and microwave systems. In this work, tunable optical delay lines based on integrated grating-assisted contradirectional couplers are proposed and experimentally demonstrated. The device performance is comprehensively improved in terms of parameter optimization, apodization analysis, and electrode design. Tunable group delay lines of 50 ps at different wavelengths within the bandwidth of $12 \mathrm{~nm}$ are realized with a grating length of $1.8 \mathrm{~mm}$. Under thermal tuning mode, the actual delay tuning range is around $20 \mathrm{ps}$ at $7.2 \mathrm{~V}$ voltage. At last, a new scheme adopting an ultra-compact reflector for doubling group delay is proposed and verified, achieving a large group delay line of $400 \mathrm{ps}$ and a large dispersion value up to $5.5 \times 10^{6} \mathrm{ps} /(\mathrm{nm} \cdot \mathrm{km})$ within bandwidth of $12 \mathrm{~nm}$. Under thermal tuning mode, the actual delay tuning range is around 100 ps at $8 \mathrm{~V}$ voltage. (๑) 2018 Chinese Laser Press
\end{abstract}

OCIS codes: (130.3120) Integrated optics devices; (130.7408) Wavelength filtering devices; (200.4490) Optical buffers.

https://doi.org/10.1364/PRJ.6.000880

\section{INTRODUCTION}

Optical delay lines have wide applications in optical communication and microwave photonics systems [1-8]. In optical communication, the main functions of optical delay lines cover signal synchronization, optical buffers, time-division multiplexing, and so forth. In microwave photonics systems, the most eye-catching application of phased array antennas highly relies on the beam-forming technology, which is usually controlled by the array of true-time delay lines.

In general, there are two approaches to the physical implementation of optical delay lines. The most direct and easiest approach for tunable group delay is changing the optical physical distance [9]. For instance, typical schemes are dependent on the selection of long or short delay paths in each stage by Mach-Zehnder interferometer switches [10-12]. Recently, a novel scheme of hexagonal integrated waveguide mesh configurations was proposed for the implementation of compact discrete and continuous optical true-time delay lines [13]. Although this approach is very intuitive and simple, the implementation of long time delays comes at the cost of large dimension, and the tunable accuracy is commonly limited by the shortest distance between switches.

The other approach to generating optical delay lines is employing resonant enhancement devices such as the microring resonators, photonic crystal waveguides (PhCWs), and Bragg gratings [14,15]. Among them, microrings are the most compact device, and can achieve continuously tunable delay. However, the delay-bandwidth product of a single microring is limited by $2 / \pi$ [16]. To increase the delay-bandwidth product, all-pass filters and coupled resonator optical waveguides with several cascaded microrings have been employed with compact structure and large delay [17-22]. In PhCWs, the strong optical confinement and slow light effects were used to realize true-time delay as well, which reduced the footprint and power consumption [23-27]. However, both of the microrings and the PhCWs suffered from high loss, which hindered the real applications. Recently, chirped Bragg gratings have been applied to realize true-time delay with low loss and wide operating band. Electrically tunable optical true-time delay lines which introduced a $\mathrm{p}-\mathrm{n}$ junction in the Bragg gratings to tune group delay at a fixed wavelength were first proposed in Ref. [28]. Ultra-compact graphene-based integrated microphotonic tunable delay lines have been designed that exhibit short optical switching time, large delay range, low loss, and weak power consumption [29]. However, these two tuning techniques are just in the theoretical design stage. Combining tapered Bragg gratings and thermo-optical effect, continuously tunable delay lines based on silicon-on-insulator (SOI) tapered Bragg gratings have also been proposed and verified experimentally [30,31]. Although tunable linear delay lines with low loss and wide band have been realized, they are still far from being ready for use in grating-assisted delay lines. 
In this paper, we propose and experimentally demonstrate tunable optical delay lines based on integrated grating-assisted contradirectional couplers. An optimized apodization method is chosen to suppress the ripples of the transmission spectrum and delay lines. Afterwards, devices with various parameters are fabricated and the impact of each parameter on the performance of the device is analyzed. To solve the problem of high heater resistance caused by the long gratings, a design of parallel electrodes is proposed that shows better heating efficiency. With the optimization methods just mentioned, tunable group delay lines of $50 \mathrm{ps}$ within the bandwidth of $12 \mathrm{~nm}$ are realized with grating length of $1.8 \mathrm{~mm}$. We combine grating-assisted contradirectional couplers with an ultra-compact reflector for double delay in the same size, and the fabricated $6 \mathrm{~mm}$ device can achieve a large group delay of $400 \mathrm{ps}$ and dispersion value of up to $5.5 \times 10^{6} \mathrm{ps} /(\mathrm{nm} \cdot \mathrm{km})$ within the bandwidth of $12 \mathrm{~nm}$.

\section{PRINCIPLE AND CHIP FABRICATION}

Figure 1 shows the scheme of the proposed integrated gratingassisted contradirectional couplers. It consists of two tapered stripe waveguides with different widths, and the Bragg gratings are formed on the sidewall of the wider one. The two port widths of the wide waveguide are $w_{1}$ and $w_{1}+\Delta w$, and the two port widths of the narrow waveguide are $w_{2}$ and $w_{2}+\Delta w$. The general chirp types of Bragg gratings are index chirping and period chirping. Index chirping is chosen here because linear variation of the effective index is more robust than varying the grating period $[30,31]$. The grating period of $\Lambda$ is kept the same along the transmission direction with duty cycle of $50 \%$. The grating number, $N$, determines the grating length $L=N \times \Lambda$. The width of the gratings $w_{g}$ and gap between the two waveguides also require optimization to introduce proper contradirectional coupling into the design.

Since the Bragg gratings are not infinite in length, the Fourier transform of the gratings with rectangle function is typically a sinc function. This means the side-lobes and ripples will appear in the transmission spectrum. Fortunately, apodization technology has been developed and widely used in mature fiber gratings to suppress side-lobes and ripples. We simulate different apodization filters to achieve a flat-top response and ripple suppression. According to the analysis in Refs. [32,33] and tentative simulations, asymmetric cosine apodization is one of the most effective methods for ripple suppressions of transmission and group delay spectra. Thus, we adopt asymmetric cosine apodization; namely, the apodization function is only applied on the gratings near the input port. The ratio of apodization length to the whole grating length actually has an effect upon the transmission and group delay spectra, as shown in Fig. 2.

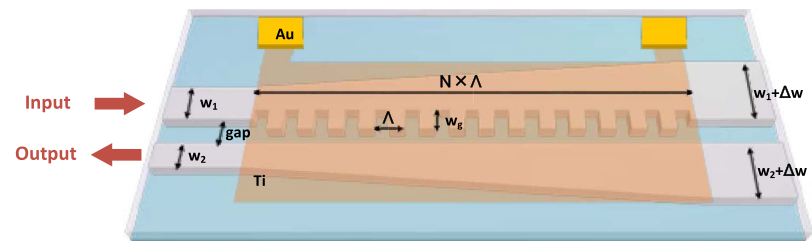

Fig. 1. Scheme of the proposed integrated grating-assisted contradirectional couplers.
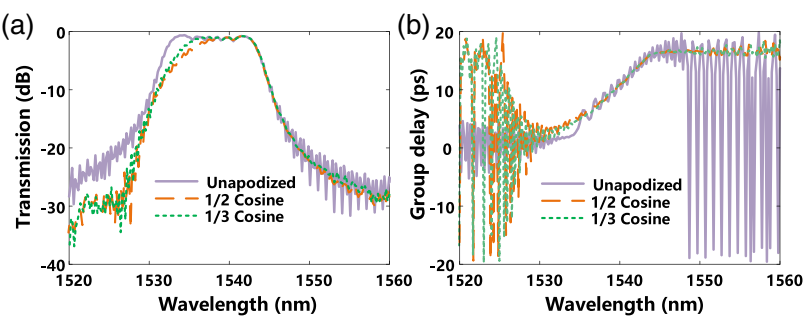

Fig. 2. (a) Simulated transmission spectra and (b) group-delay spectra for chirped Bragg gratings with different apodization filters applied. $L=710.4 \mu \mathrm{m}, w_{g}=50 \mathrm{~nm}, w_{1}=570 \mathrm{~nm}, w_{2}=470 \mathrm{~nm}$, $\Delta w=20 \mathrm{~nm}$, gap $=180 \mathrm{~nm}$.

The Bragg gratings are chosen with $L=710.4 \mu \mathrm{m}, w_{g}=$ $50 \mathrm{~nm}, w_{1}=570 \mathrm{~nm}, w_{2}=470 \mathrm{~nm}, \Delta w=20 \mathrm{~nm}$, and gap $=180 \mathrm{~nm}$. Figure 2(a) shows that the ripples of the transmission spectra are reduced when asymmetric cosine apodization is applied. In addition, the bandwidth loss of $1 / 3$ cosine apodization is lower than that of $1 / 2$ cosine apodization. In Fig. 2(b), the ripples of group-delay spectra are well suppressed when asymmetric cosine apodization of either $1 / 3$ or $1 / 2$ cosine is applied. Ultimately, $1 / 3$ cosine apodization is selected due to its superior performance.

The central wavelength of the Bragg gratings follows the Bragg equation,

$$
\lambda_{c}=2 n_{\mathrm{eff}} \Lambda \text {. }
$$

Here, $n_{\text {eff }}$ and $\Lambda$ are determined by the device, so devices with various parameters are fabricated to investigate the effect on the transmission spectrum. The lengths of the fabricated devices are all about $1.8 \mathrm{~mm}$, with $N=6000$. Figure 3(a) shows the measured transmission spectra for different chip samples. One can see that the transmission spectra are redshifted with increasing grating period, which agrees with the equation. In Fig. 3(b), the waveguide widths are designed as $w_{1}=600 \mathrm{~nm}$ and $w_{2}=500 \mathrm{~nm}$. With wider waveguides, the effective index of the waveguide increases, which results in the redshifting of all the transmission spectra. In addition, the bandwidth of the wider waveguides is about $9 \mathrm{~nm}$, which is smaller than that of the narrower waveguides. Next, chirp rate is varied by different $\Delta w$ of $10 \mathrm{~nm}$ and $20 \mathrm{~nm}$. Figure 3(c) shows that large chirp rate results in a wider reflection bandwidth, i.e., 6.8 versus $12 \mathrm{~nm}$. The measured results in Figs. 3(a)-3(c) are all based on unapodized gratings. In Fig. 3(d), unapodized and asymmetric apodized devices are compared, and it should be noted that the passband spectra of the gratings are smoother thanks to the apodization design.

The proposed structure is fabricated on a commercial SOI wafer which consists of a $250 \mathrm{~nm}$ silicon layer and a $3 \mu \mathrm{m}$ buried oxide layer. First, the waveguide patterns are defined by electron beam lithography (EBL) on the commercial SOI wafer, and then the silicon is dry etched by the inductively coupled plasma etching process. Second, a $2 \mu \mathrm{m}$ thick silicon dioxide layer is deposited using plasma-enhanced chemical vapor deposition (PECVD). Finally, the heaters are formed by depositing metal layers on the $\mathrm{SiO}_{2}$ layer. Figures 3(e) and 3(f) show the scanning electron microscopy (SEM) micrographs of fabricated apodized and unapodized structures before 

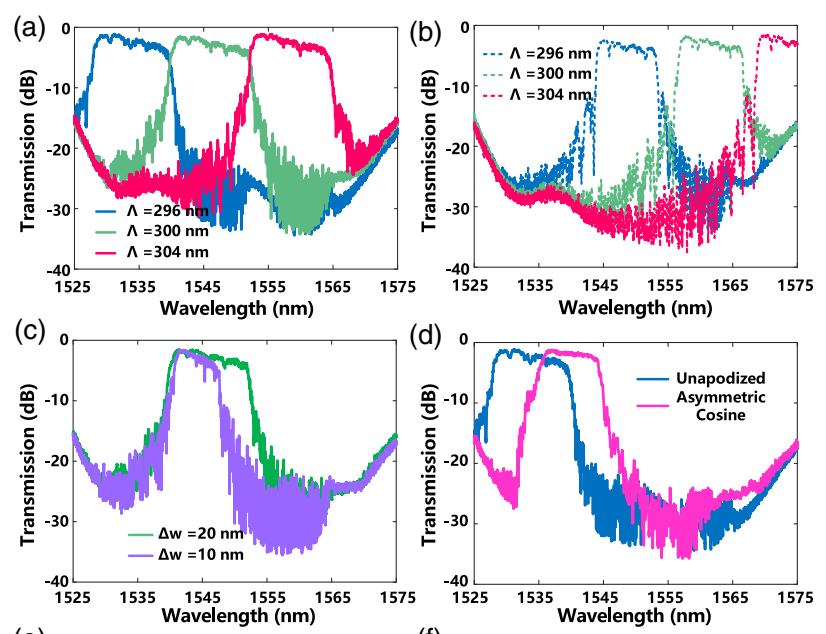

(e)
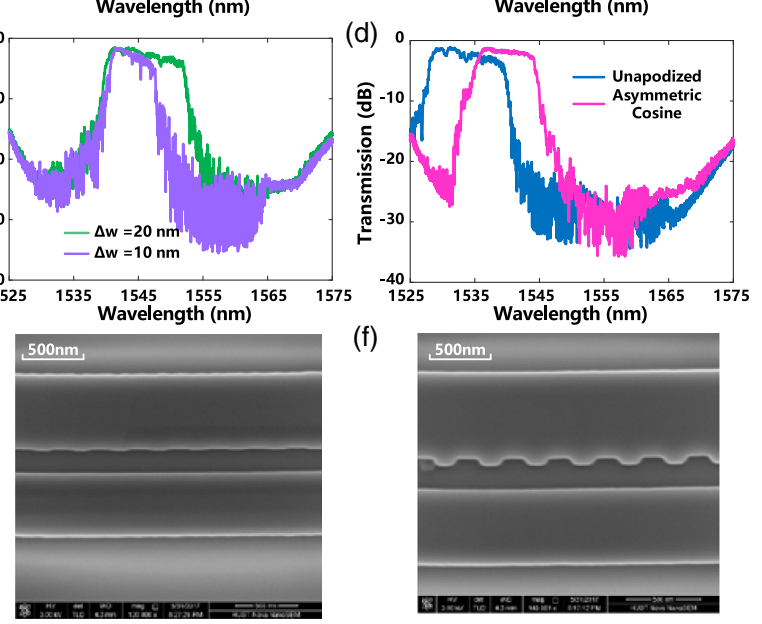

(f)

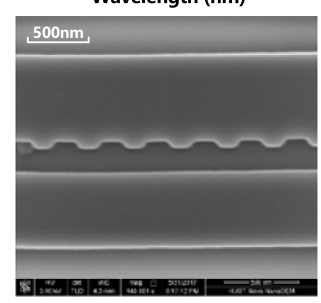

Fig. 3. (a) Measured transmission spectra of different grating periods with $w_{1}=570 \mathrm{~nm}, w_{2}=470 \mathrm{~nm}$, and $\Delta w=20 \mathrm{~nm}$. (b) Measured transmission spectra of different grating periods with $w_{1}=600 \mathrm{~nm}$ and $w_{2}=500 \mathrm{~nm}$. (c) Measured transmission spectra of different $\Delta w$ with $w_{1}=570 \mathrm{~nm}, w_{2}=470 \mathrm{~nm}$, and $\Lambda=$ $296 \mathrm{~nm}$. (d) Measured transmission spectra of unapodized and asymmetric apodized Bragg gratings with $w_{1}=570 \mathrm{~nm}, w_{2}=470 \mathrm{~nm}$, $\Lambda=296 \mathrm{~nm}$, and $\Delta w=20 \mathrm{~nm}$. (e) SEM image of apodized Bragg gratings. (d) SEM image of unapodized Bragg gratings.

PECVD deposition. It should be noted that the fabrication process requires high resolution of EBL to achieve slight amplitude modulation along the waveguide.

The tunability of time delay lines is achieved by the thermooptic effect of the heating electrodes. A $100 \mathrm{~nm}$ thick titanium strip is deposited on top of the oxide cladding for thermal tuning, and $10 \mathrm{~nm}$ thick gold is deposited as conductive electrode. However, due to the millimeter-scale length of the Bragg gratings, the fabricated heating metal layer length is also millimeter-scale, with a large resistance. For example, the resistance of the strip heaters (type I) in Fig. 4(a) is measured as $12 \mathrm{k} \Omega$ with a $1.71 \mu \mathrm{m}$ wide, $1.8 \mathrm{~mm}$ long titanium strip. In this case, the spectrum drift is only $0.3 \mathrm{~nm}$, and even the applied voltage is up to $20 \mathrm{~V}$. To enhance the heating efficiency, heaters with parallel circuits (type II) are designed to reduce the resistance. The resistance of the type II heaters in Fig. 4(a) is measured as $70 \Omega$ with a $3 \mu \mathrm{m}$ wide, $100 \mathrm{~nm}$ thick, $1.8 \mathrm{~mm}$ long titanium strip. As shown in Fig. 4(b), type II heaters can achieve $4.68 \mathrm{~nm}$ spectrum drift when the applied voltage is $8 \mathrm{~V}$. The heating efficiency is enhanced by about 94 times at $8 \mathrm{~V}$ voltage. The micrographs of the type I and type II devices are shown in Figs. 4(c) and 4(d), respectively. In this part, we propose a type II heater to improve heating efficiency for narrow and long waveguides. Of course, this is not the only way to reduce resistance; an alternative way is to increase the thickness and (a)

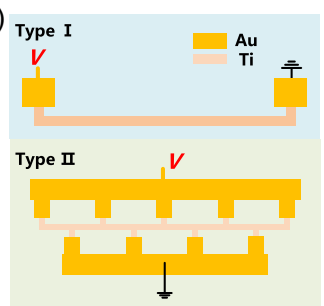

(c)

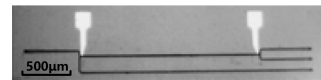

(b)

(d)


Fig. 4. (a) Schematic of type I and type II heaters. (b) Spectral drift as a function of the applied voltage of the two types of heaters. Micrographs of (c) type I and (d) type II heaters.

width of the heaters. However, the large width of the heaters will lead to a lower heating efficiency. Furthermore, increasing the thickness will be more expensive, and sometimes the thickness is limited by the standard process flow. The type II design offers the possibility of implementing a high-efficiency metal microheater by simply changing the design on the planar layer.

\section{RESULTS AND DISCUSSION}

The experimental setup for the measurement of group delay is shown in Fig. 5. The optical carrier is provided by a tunable laser source (TLS) with $+10 \mathrm{dBm}$ optical power. Then the launched light passes through the polarization controller (PC1) and $10 \mathrm{GHz}$ intensity modulator (IM). The $10 \mathrm{GHz}$ radio frequency $(R F)$ signal is loaded on the IM through the RF amplifier. Afterwards, a $10 \mathrm{GHz}$ modulated sinusoidal signal is created. The signal is then injected into the proposed device via $\mathrm{PC} 2$, and is finally detected by an oscilloscope (OSC). The PCs are used to maximize the efficiency of mode coupling. The two erbium-doped fiber amplifiers are used to compensate link loss, and the attenuator is used to protect the OSC. The fiber-to-fiber insertion loss is about $6 \mathrm{~dB}$. When the input wavelength changes within the passband, the detected waveforms will have different time delays. By comparing the time delay at different wavelengths, the group delay lines can be calculated.

There are two modes for delay tuning using optical delay lines. One mode is to tune the time delay by changing the optical carrier wavelength. However, this tuning mode relies

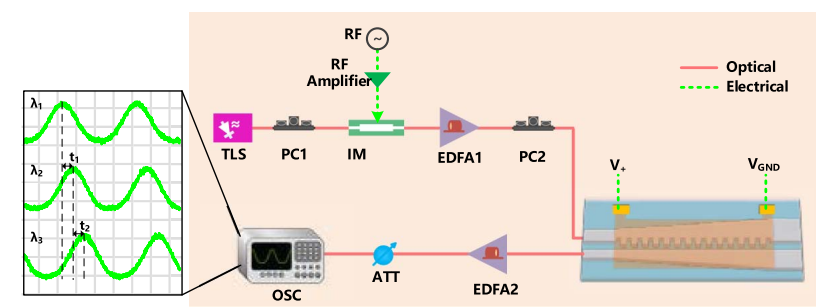

Fig. 5. Experimental setup of the time-delay measurement. TLS, tunable laser source; PC, polarization controller; RF, radio frequency; IM, intensity modulator; EDFA, erbium-doped fiber amplifier; ATT, attenuator; OSC, oscilloscope. 
on a laser source with a tunable wavelength. In most cases, the laser source has a fixed wavelength without tunability. Another mode is to tune the time delay by the drift of dispersion curve driven by the loaded voltage. In this case, the laser source does not need to tune its wavelength. To prevent the breakdown of the metal microheater, the driving voltage is limited to less than $8 \mathrm{~V}$, thus restraining the maximum delay tuning range. This can be further improved by optimizing electrode thickness and resistance characteristics. In the measurement that follows, the two cases both will be discussed.

Figure 6 shows the measured spectral responses and group delay lines of unapodized and asymmetric apodized fabricated devices of $1.8 \mathrm{~mm}$ length. In the absence of voltage, the passband of the unapodized device in Fig. 6(a) is $12 \mathrm{~nm}$. The insertion loss near the band edge at the short-wavelength side $(1541.4 \mathrm{~nm})$ and long-wavelength side $(1552 \mathrm{~nm})$ are 0.5 and $2.7 \mathrm{~dB}$, respectively. In Fig. 6(b), the passband of the apodized device is $8 \mathrm{~nm}$. The insertion loss near the band edge at the short-wavelength side $(1536.5 \mathrm{~nm})$ and long-wavelength side $(1544.2 \mathrm{~nm})$ are 1.33 and $2.6 \mathrm{~dB}$, respectively. It can be seen that the passband of the apodized device is smoother than that of the unapodized one, and both spectra can maintain the same shape under different voltages of $0,5.2$, and $7.2 \mathrm{~V}$. We also notice that the apodization method brings ripple suppression at the cost of bandwidth reduction and delay loss. In Fig. 6(c), the total time delay of the unapodized device in the passband is about 55 ps, and when the device is operating at heating status, the group delay lines have large fluctuations. In Fig. 6(d), the total delay of the apodized device is reduced to $50 \mathrm{ps}$, but both the linearity and consistency have been improved. A figure of merit to evaluate the delay line performance is the loss per ns delay increment (in $\mathrm{dB} / \mathrm{ns}$ unit). This parameter is 40 and $25.4 \mathrm{~dB} / \mathrm{ns}$ for the unapodized and apodized device, respectively. Compared to the parameter in Refs. [30] (9.6 dB/ns) and [31] $(21 \mathrm{~dB} / \mathrm{ns})$, the device loss can be further reduced by waveguide mode optimization. In conclusion, the apodization method can improve the performance of Bragg gratings in terms of spectral flatness, delay line linearity, and optical loss.
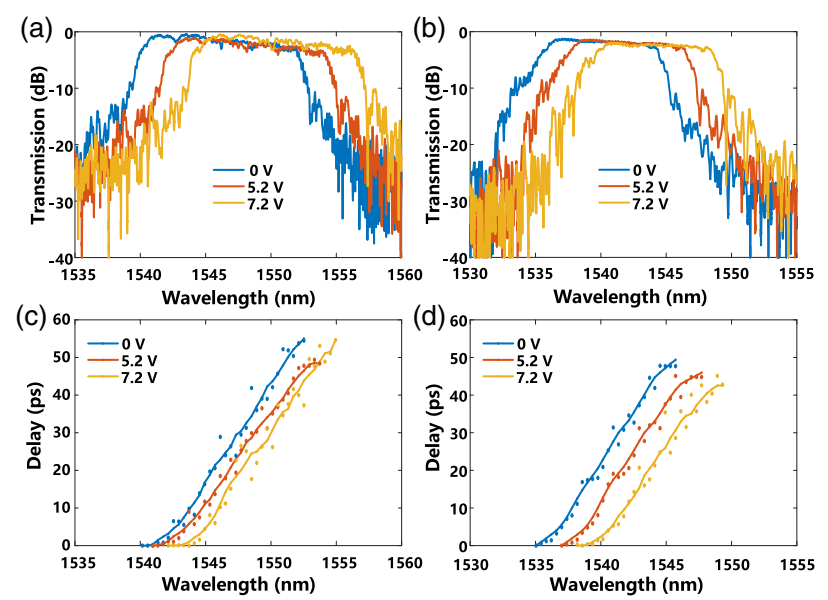

Fig. 6. Measured spectral responses of (a) unapodized and (b) asymmetric apodized fabricated devices. Measured group delay lines of (c) unapodized and (d) asymmetric apodized fabricated devices.
When the laser wavelength is scanned within the passband of the gratings, the output optical signals will have different delay values. To demonstrate the delay tunability, we measured the output waveforms with different wavelengths after propagating in the apodized device, as shown in Fig. 7. The gray dot curves and red curves in Fig. 7 represent the $10 \mathrm{GHz}$ input sine waveforms and delayed waveforms, respectively. By changing the optical carrier wavelength from 1535 to $1545 \mathrm{~nm}$, the delay tuning range is about 50 ps. In addition, there is no distortion in the output waveforms at a modulated bandwidth of $10 \mathrm{GHz}$. Under thermal tuning mode, the actual delay tuning range for the devices is around $20 \mathrm{ps}$ at $7.2 \mathrm{~V}$.

For the schemes based on chirped Bragg gratings, the maximum group delay is limited by the grating length. To improve the group delay of a certain length, we propose a method to combine the scheme with an ultra-compact reflector [34] for doubling group delay, as shown in Fig. 8(a). The reflector is set at the original output port of the contradirectional couplers, so the light of different wavelengths can return to the input port through different paths. A Y-branch is introduced at the input port, so it introduces an extra loss of about $6 \mathrm{~dB}$. This method improves the utilization of the Bragg gratings. It should be noted that the use of a reflector can obtain a doubled delay amount, but an insertion loss of about $6 \mathrm{~dB}$ is introduced by the Y-branch at the input port. The use of a Y-branch is convenient for measurement, and it can be removed in the practical application. Despite the Y-branch, the insertion losses are almost the same compared to a grating of double length, and the proposed device has more compact size. In the future, we can reduce the chip size with a spiral pattern design $[35,36]$.

The micrograph of the fabricated device with $6 \mathrm{~mm}$ long Bragg gratings is shown in Fig. 8(d). The reflector has a compact size of $5 \mu \mathrm{m} \times 5 \mu \mathrm{m}$, which has almost no impact on the

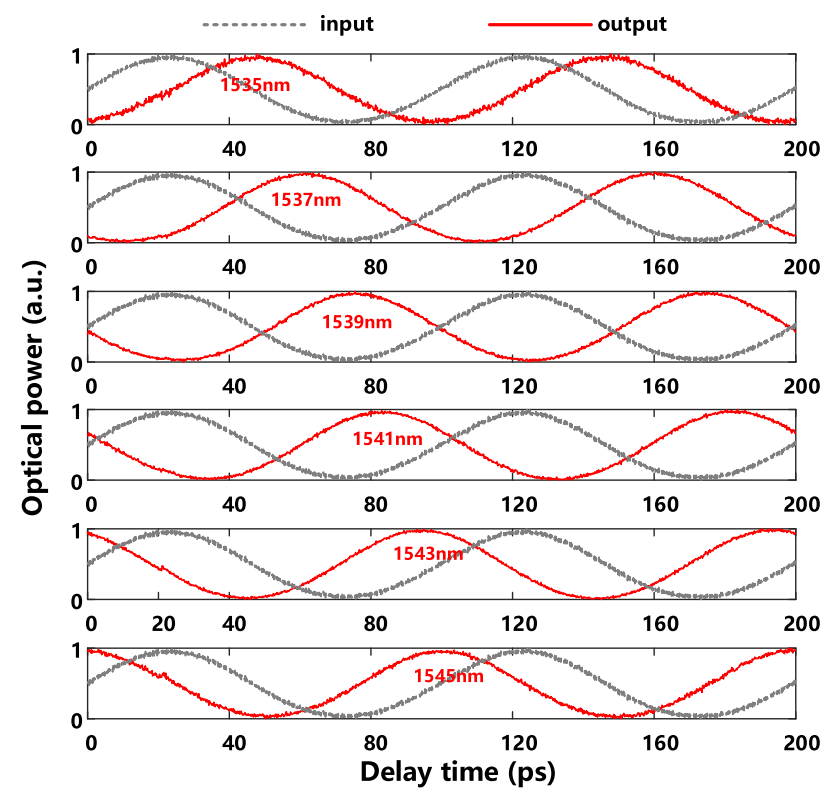

Fig. 7. $10 \mathrm{GHz}$ optical waveforms with different wavelengths after passing through the apodized device. Gray dot curves represent input waveforms; red curves represent delayed waveforms. 
(a)

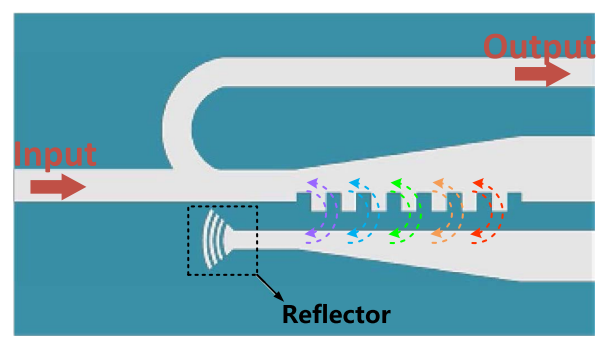

(d)

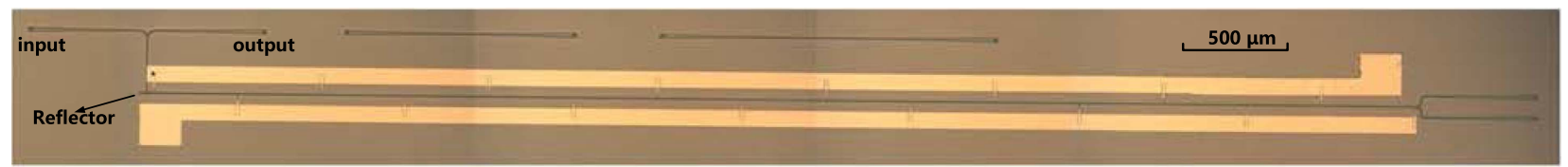

Fig. 8. (a) Schematic of a novel structure combining the grating-assisted contradirectional couplers with an ultra-compact reflector. (b) Measured spectral responses and (c) group delay lines of the fabricated device at different voltages. (d) Micrograph of the fabricated device.

overall device size. The resistance of type II heaters in Fig. 8(d) is measured as $60 \Omega$ with a $3 \mu \mathrm{m}$ wide, $200 \mathrm{~nm}$ thick, $6 \mathrm{~mm}$ long titanium strip. The measured spectral responses and group delay lines of the fabricated device are shown in Figs. 8(b) and 8(c). In Fig. 8(b), the passband of the device is $12 \mathrm{~nm}$. The insertion loss near the band edge at the short-wavelength side $(1536.5 \mathrm{~nm})$ and long-wavelength side $(1549 \mathrm{~nm})$ are 7 and $15 \mathrm{~dB}$, respectively. In the group delay measurement, a $5 \mathrm{GHz}$ modulated sinusoidal signal is employed as the test signal. In Fig. 8(c), the measured group delay can achieve 400 ps within the passband of $12 \mathrm{~nm}$. The delay lines have a slope of $33 \mathrm{ps} / \mathrm{nm}$, indicating a positive chromatic dispersion of $5.5 \times 10^{6} \mathrm{ps} /(\mathrm{nm} \cdot \mathrm{km})$, which is enhanced by $3.2 \times 10^{5}$ times compared to the dispersion of single-mode fiber with $17 \mathrm{ps} /(\mathrm{nm} \cdot \mathrm{km})$ dispersion. Compared to recent research about dispersion engineering, our chip design may pave the way for on-chip large-dispersion units with a compact size [37-39]. With increasing applied voltage, a redshift occurs in both the spectral responses and group delay lines. However, due to the reflection between the Y-branch and reflector, there are some ripples in the transmission spectra, and the transmission has a larger attenuation at the longer wavelength. When the applied voltage is up to $8 \mathrm{~V}$, distortion occurs in the group delay lines. Due to the long waveguide length, the waveguide and heater distribution cannot be completely uniform in the propagation direction. Since the waveguide is heated unevenly, the transmission spectrum resonates at some wavelengths, resulting in a sudden change in phase, so the delay at some wavelengths fluctuates. Further optimizations are required to avoid resonance among the device.

Due to the large group delay fluctuations and dispersion of the grating-assisted contradirectional couplers with an ultracompact reflector, we use a narrow-bandwidth optical signal of $5 \mathrm{GHz}$ to evaluate the performance of the device. The gray dot curves and red curves in Fig. 9 represent the $5 \mathrm{GHz}$ input sine waveforms and delayed waveforms, respectively. With the input wavelength increasing from 1536 to $1550 \mathrm{~nm}, 400$ ps delay tuning range can be achieved. We can see that the delayed waveform at $1540 \mathrm{~nm}$ has slight distortion, which is caused by

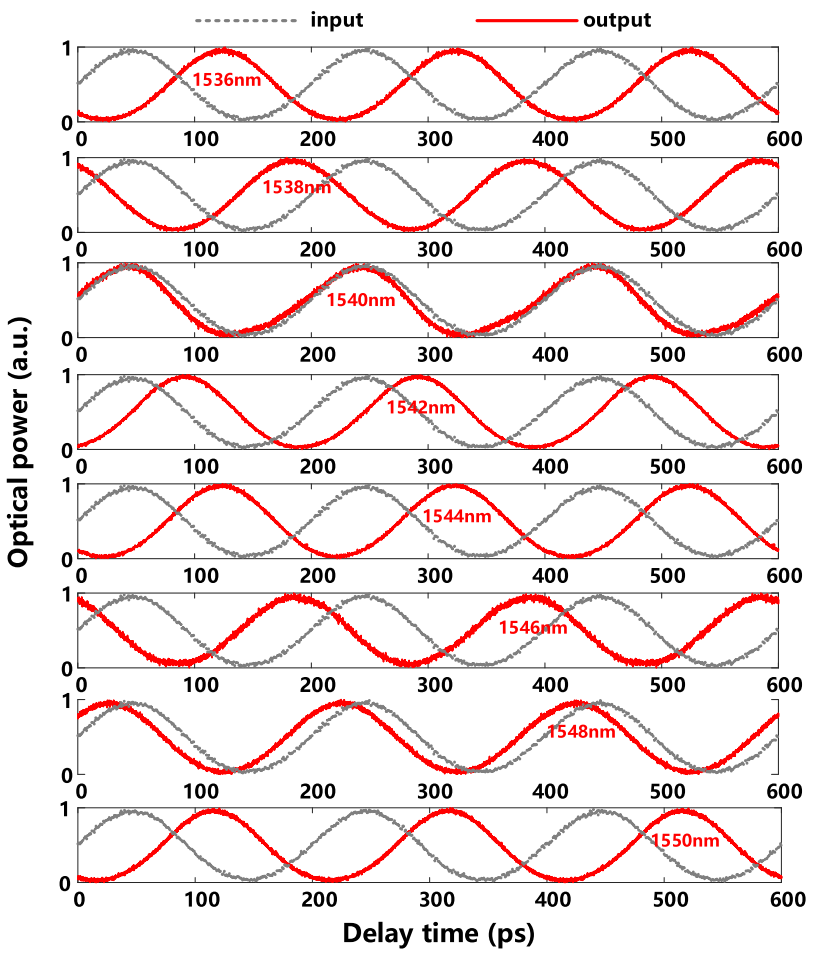

Fig. 9. $5 \mathrm{GHz}$ optical waveforms with different wavelengths after passing through the gratings with an ultra-compact reflector. Gray dot curves represent input waveforms; red curves represent delayed waveforms.

the group delay fluctuations. In general, the output waveform can maintain sine waveform within the passband.

\section{CONCLUSION}

To summarize, we propose and demonstrate tunable optical delay lines based on integrated grating-assisted contradirectional couplers. We studied and optimized device performance 
from several aspects, including parameter optimization, apodization analysis, and electrode and large delay design. Finally, tunable group delay lines of 50 ps at different wavelengths within the bandwidth of $12 \mathrm{~nm}$ are realized with an apodized Bragg grating length of $1.8 \mathrm{~mm}$. The delay tuning range under thermal tuning mode is about 20 ps, and it maintains good linearity and consistency at different voltages. In addition, a novel structure combining the grating-assisted contradirectional couplers with an ultra-compact reflector is discussed and a large group delay of 400 ps within the bandwidth of $12 \mathrm{~nm}$ is achieved. The delay tuning range under the thermal tuning mode is about 100 ps. A dispersion value up to $5.5 \times$ $10^{6} \mathrm{ps} /(\mathrm{nm} \cdot \mathrm{km})$ with bandwidth of $12 \mathrm{~nm}$ is demonstrated in the communication band. Our work paves the way for onchip tunable delay lines and large-dispersion devices.

Funding. National Natural Science Foundation of China (NSFC) (61475052, 61622502); Fundamental Research Funds for the Central Universities (2017KFXKJC001).

\section{REFERENCES}

1. F. Xia, L. Sekaric, and Y. Vlasov, "Ultracompact optical buffers on a silicon chip," Nat. Photonics 1, 65-71 (2007).

2. M. Moralis-Pegios, N. Terzenidis, G. Mourgias-Alexandris, K. Vyrsokinos, and N. Pleros, "A low-latency high-port count optical switch with optical delay line buffering for disaggregated data centers," Proc. SPIE 10538, 1053805 (2018).

3. J. L. Corral, J. Marti, J. M. Fuster, and R. I. Laming, "True time-delay scheme for feeding optically controlled phased-array antennas using chirped-fiber gratings," IEEE Photon. Technol. Lett. 9, 1529-1531 (1997).

4. C. R. Doerr, S. Chandrasekhar, P. J. Winzer, A. R. Chraplyvy, A. H. Gnauck, L. W. Stulz, R. Pafchek, and E. Burrows, "Simple multichannel optical equalizer mitigating intersymbol interference for $40-\mathrm{Gb} / \mathrm{s}$ nonreturn-to-zero signals," J. Lightwave Technol. 22, 249-256 (2004).

5. F. Wang and X. Zhang, "Photonic generation of ultrawideband signals using a delay interferometer," Front. Optoelectron. China 3, 179-183 (2010).

6. Z. $\mathrm{Hu}, \mathrm{J} . \mathrm{Xu}$, and M. Hou, "Theoretical demonstration of all-optical switchable and tunable UWB doublet pulse train generator utilizing SOA wavelength conversion and tunable time delay," Front. Optoelectron. 10, 180-188 (2017).

7. R. L. Moreira, J. Garcia, W. Li, J. Bauters, J. S. Barton, M. J. R. Heck, J. E. Bowers, and D. J. Blumenthal, "Integrated ultra-low-loss 4-bit tunable delay for broadband phased array antenna applications," IEEE Photon. Technol. Lett. 25, 1165-1168 (2013).

8. V. C. Duarte, J. G. Prata, C. Ribeiro, R. N. Nogueira, G. Winzer, L. Zimmermann, R. Walker, S. Clements, M. Filipowicz, M. Napierała, T. Nasiłowski, J. Crabb, L. Stampoulidis, J. Anzalchi, and M. V. Drummond, "Integrated photonic true-time delay beamformer for a Ka-band phased array antenna receiver," in Optical Fiber Communication Conference, OSA Technical Digest (online) (Optical Society of America, 2018), paper M2G.5.

9. H. Lee, T. Chen, J. Li, O. Painter, and K. J. Vahala, "Ultra-low-loss optical delay line on a silicon chip," Nat. Commun. 3, 867 (2012).

10. X. Wang, B. Howley, M. Y. Chen, and R. T. Chen, "Phase error corrected 4-bit true time delay module using a cascaded $2 \times 2$ polymer waveguide switch array," Appl. Opt. 46, 379-383 (2007).

11. J. Xie, L. Zhou, Z. Li, J. Wang, and J. Chen, "Seven-bit reconfigurable optical true time delay line based on silicon integration," Opt. Express 22, 22707-22715 (2014)

12. X. Wang, L. Zhou, R. Li, J. Xie, L. Lu, K. Wu, and J. Chen, "Continuously tunable ultra-thin silicon waveguide optical delay line," Optica 4, 507-515 (2017).
13. D. Perez, E. S. Gomariz, and J. Capmany, "Programmable true-time delay lines using integrated waveguide meshes," J. Lightwave Technol. PP, 1 (2018).

14. R. Kashyap and M. de Lacerda Rocha, "On the group delay characteristics of chirped fibre Bragg gratings," Opt. Commun. 153, 19-22 (1998).

15. G. Lenz, B. J. Eggleton, C. K. Madsen, and R. E. Slusher, "Optical delay lines based on optical filters," IEEE J. Quantum Electron. 37, 525-532 (2001).

16. L. Y. Mario and M. K. Chin, "Optical buffer with higher delay-bandwidth product in a two-ring system," Opt. Express 16, 1796-1807 (2008).

17. M. S. Rasras, C. K. Madsen, M. A. Cappuzzo, E. Chen, L. T. Gomez, E. J. Laskowski, A. Griffin, A. Wong-Foy, A. Gasparyan, A. Kasper, J. L. Grange, and S. S. Patel, "Integrated resonance-enhanced variable optical delay lines," IEEE Photon. Technol. Lett. 17, 834-836 (2005).

18. F. Morichetti, A. Melloni, A. Breda, A. Canciamilla, C. Ferrari, and M. Martinelli, "A reconfigurable architecture for continuously variable optical slow-wave delay lines," Opt. Express 15, 17273-17282 (2007).

19. A. Melloni, F. Morichetti, C. Ferrari, and M. Martinelli, "Continuously tunable 1 byte delay in coupled-resonator optical waveguides," Opt. Lett. 33, 2389-2391 (2008).

20. F. Morichetti, A. Melloni, C. Ferrari, and M. Martinelli, "Error-free continuously-tunable delay at $10 \mathrm{Gbit} / \mathrm{s}$ in a reconfigurable on-chip delay-line," Opt. Express 16, 8395-8405 (2008).

21. J. Xie, L. Zhou, Z. Zou, J. Wang, X. Li, and J. Chen, "Continuously tunable reflective-type optical delay lines using microring resonators," Opt. Express 22, 817-823 (2014).

22. C. Xiang, M. L. Davenport, J. B. Khurgin, P. A. Morton, and J. E. Bowers, "Low-loss continuously tunable optical true time delay based on $\mathrm{Si}_{3} \mathrm{~N}_{4}$ ring resonators," IEEE J. Sel. Top. Quantum Electron. 24, 1-9 (2018).

23. J. Li, T. P. White, L. O'Faolain, A. Gomez-Iglesias, and T. F. Krauss, "Systematic design of flat band slow light in photonic crystal waveguides," Opt. Express 16, 6227-6232 (2008).

24. J. Adachi, N. Ishikura, H. Sasaki, and T. Baba, "Wide range tuning of slow light pulse in SOI photonic crystal coupled waveguide via folded chirping," IEEE J. Sel. Top. Quantum Electron. 16, 192-199 (2010).

25. A. Melloni, A. Canciamilla, C. Ferrari, F. Morichetti, L. O. Faolain, T. F. Krauss, R. D. L. Rue, A. Samarelli, and M. Sorel, "Tunable delay lines in silicon photonics: coupled resonators and photonic crystals, a comparison," IEEE Photon. J. 2, 181-194 (2010).

26. C.-Y. Lin, H. Subbaraman, A. Hosseini, A. X. Wang, L. Zhu, and R. T. Chen, "Silicon nanomembrane based photonic crystal waveguide array for wavelength-tunable true-time-delay lines," Appl. Phys. Lett. 101, 051101 (2012)

27. C.-J. Chung, X. Xu, G. Wang, Z. Pan, and R. T. Chen, "On-chip optical true time delay lines featuring one-dimensional fishbone photonic crystal waveguide," Appl. Phys. Lett. 112, 071104 (2018).

28. S. Khan, M. A. Baghban, and S. Fathpour, "Electronically tunable silicon photonic delay lines," Opt. Express 19, 11780-11785 (2011).

29. G. Brunetti, D. Conteduca, F. Dell'Olio, C. Ciminelli, and M. N. Armenise, "Design of an ultra-compact graphene-based integrated microphotonic tunable delay line," Opt. Express 26, 4593-4604 (2018).

30. I. Giuntoni, D. Stolarek, D. I. Kroushkov, J. Bruns, L. Zimmermann, B. Tillack, and K. Petermann, "Continuously tunable delay line based on SOI tapered Bragg gratings," Opt. Express 20, 11241-11246 (2012).

31. W. Shi, V. Veerasubramanian, D. Patel, and D. V. Plant, "Tunable nanophotonic delay lines using linearly chirped contradirectional couplers with uniform Bragg gratings," Opt. Lett. 39, 701-703 (2014).

32. D. Tan, K. Ikeda, R. Saperstein, B. Slutsky, and Y. Fainman, "Chipscale dispersion engineering using chirped vertical gratings," Opt. Lett. 33, 3013-3015 (2008).

33. R. Kashyap, Fiber Bragg Gratings (Academic, 1999).

34. Y. Wang, S. Gao, K. Wang, H. Li, and E. Skafidas, "Ultra-broadband, compact, and high-reflectivity circular Bragg grating mirror based on 220 nm silicon-on-insulator platform," Opt. Express 25, 6653-6663 (2017).

35. Z. Chen, J. Flueckiger, X. Wang, F. Zhang, H. Yun, Z. Lu, M. Caverley, Y. Wang, N. A. Jaeger, and L. Chrostowski, "Spiral Bragg grating 
waveguides for TM mode silicon photonics," Opt. Express 23, 2529525307 (2015).

36. Z. Zou, L. Zhou, M. Wang, K. Wu, and J. Chen, "Tunable spiral Bragg gratings in 60-nm-thick silicon-on-insulator strip waveguides," Opt. Express 24, 12831-12839 (2016).

37. D. T. H. Tan, K. Ikeda, R. E. Saperstein, B. Slutsky, and Y. Fainman, "Chip-scale dispersion engineering using chirped vertical gratings," Opt. Lett. 33, 3013-3015 (2008).
38. E. Sahin, K. J. A. Ooi, C. E. Png, and D. T. H. Tan, "Large on-chip dispersion using cladding-modulated 1D photonic crystals," in Opto-Electronics and Communications Conference (OECC) and Photonics Global Conference (PGC) (2017), pp. 1-3.

39. E. Sahin, K. J. A. Ooi, C. E. Png, and D. T. H. Tan, "Large, scalable dispersion engineering using cladding-modulated Bragg gratings on a silicon chip," Appl. Phys. Lett. 110, 161113 (2017). 